\title{
THE EFFECT OF CLOTHING ON THE ABILITY OF MEN TO WORK IN INTENSE HEAT
}

\author{
By WALTER B. SHELLEY,1 LUDWIG W. EICHNA,2 AND STEVEN M. HORVATH 3 \\ (From the Armored Medical Research Laboratory, Fort Knox, Kentucky)
}

(Received for publication February 7, 1946)

Although many observations have been made on the upper limits of heat tolerated by working men (1 to 6 ), little work has been published on the specific effects of clothing on man's performance in these limiting hot environments. Gagge, Winslow, and Herrington's calorimetric studies (7) on the effect of clothing were done on resting men in environments with operative temperatures below $101^{\circ} \mathrm{F}$. However, Robinson, Turrell, and Gerking (8) have studied this problem by comparing the most severe environments in which both nude and clothed men could maintain thermal equilibrium after the second hour of a six hour work period. They showed experimentally that men wearing a single layer of light clothing (windbreak poplin) could maintain thermal equilibrium only when the environments were less severe than those tolerated by nude men. The clothing had the same effect as lowering the limiting wet bulb temperature of the environment $2^{\circ}$ to $6^{\circ} \mathrm{F}$., depending on the work rate and environment.

A previous report from this laboratory (6) described the upper limits of heat that could be tolerated by highly acclimatized nude men working for four hours at approximately $250 \mathrm{Cal}$. per hour. The present investigation was undertaken to extend these data and to study the rôle of clothing by determining certain of the most severe environments in which highly acclimatized clothed men could work at this rate.

\section{PROCEDURE}

All experiments were conducted during January, February and March 1945 in a laboratory hot room $\left(35^{\prime} \times\right.$ $\left.22^{\prime} \times 14^{\prime}\right)$. A Carrier 15-T6 air processing unit permitted control to within + or $-1^{\circ} \mathrm{F}$. of the desired dry or wet bulb air temperature. For each set of conditions the air temperature was maintained for four to five days before any tests were conducted so that walls and air were

1 Captain, M.C., A.U.S.

2 Major, M.C., A.U.S.

3 Major, Sn.C., A.U.S. in equilibrium. Throughout the tests, the dry and wet bulb temperatures were measured every fifteen minutes, by means of motor driven psychrometers carried around the track at a level of four feet. Air temperatures showed a gradient of about 4 degrees from the floor to the six foot level. Wall temperatures were approximately $2^{\circ}$ to $5^{\circ}$ cooler than the air, floor temperatures $10^{\circ}$ cooler at a dry bulb temperature of $95^{\circ} \mathrm{F}$. and up to $20^{\circ}$ cooler at $120^{\circ} \mathrm{F}$. The air movement was turbulent and was essentially that produced by the movement of the men marching at $3 \mathrm{mph}$.

In the zone of hot environments in which the dry bulb temperature (D.B.) ranges from $90^{\circ}$ to $120^{\circ} \mathrm{F}$. man's performance is limited by the wet bulb temperature (W.B.) (6). Accordingly, it was felt that the upper limits of working men's tolerance to heat could be determined by studying man's ability to march for four hours under varying wet bulb temperatures at two levels of dry bulb temperature. After preliminary study the following test environments were chosen because they represent critical wet bulb temperatures.

\begin{tabular}{r|c|c}
\hline \hline $\begin{array}{c}\text { D.B. } \\
(\circ \mathbf{F})\end{array}$ & $\begin{array}{c}\text { W.B. } \\
(\circ \mathbf{F})\end{array}$ & $\begin{array}{c}\text { R.H. } \\
\text { (per cent) }\end{array}$ \\
\hline 95 & 94 & 97 \\
93 & 92 & 97 \\
120 & 92 & 35 \\
120 & 90 & 31 \\
120 & 88 & 25 \\
\hline
\end{tabular}

Ten healthy male soldiers (Table I) were the subjects. After a two-week physical conditioning program in the cool, the men were acclimatized to the heat by graded

TABLE I

Physical characteristics of subjects

\begin{tabular}{c|c|c|c|c}
\hline \hline Name & Age & Weight & Height & Surface area \\
\cline { 2 - 3 } & yrs. & lbs. & in. & $M^{2}$ \\
Di & 21 & 140 & 68 & 1.75 \\
Hi & 24 & 150 & 67 & 1.78 \\
Ka & 24 & 171 & 71 & 1.97 \\
Kn & 20 & 168 & 72 & 1.99 \\
Li & 20 & 169 & 72 & 1.99 \\
Lo & 20 & 141 & 64 & 1.69 \\
Ma & 20 & 153 & 70 & 1.86 \\
Mi & 20 & 145 & 69 & 1.80 \\
Sc & 21 & 178 & 71 & 2.02 \\
Sz & 23 & 144 & 69 & 1.80 \\
\hline Mean & 21 & 156 & 69 & 1.87 \\
\hline
\end{tabular}


activity at D.B. $120^{\circ}-$ W.B. $88^{\circ}$ F. for 21 days before the test began. They were fully acclimatized to four hours of marching with pack in this environment before any of the test environments were studied. In addition to this preliminary acclimatization, the men marched for 3 days in each new environment, before the testing was done. This added acclimatization to each specific environment was necessary to insure peak performance at these limiting environments which did impose a greater thermal load than D.B. $120^{\circ}-$ W.B. $88^{\circ}$ F. At each environment the nude and clothed state were compared in each man on two consecutive test days. The standard work consisted of marching with $20 \mathrm{lb}$. packs, at $3 \mathrm{mph}$. for four hours continuously around a 77 foot track in the hot room. This work rate was previously determined on other subjects to be approximately $250 \mathrm{Cal}$. per hour. The four work periods each morning were followed by a three hour rest in the nude, in the afternoon. At night the men lived in barracks maintained at about $70^{\circ} \mathrm{F}$.

Each man was studied (1) in the nude, wearing only shoes and socks, (2) clothed in a standard two piece single layer herringbone twill $(8 \mathrm{oz}$.) army fatigue uniform, and (3) clothed in a special impregnated-herringbone twill uniform. This latter garment was treated with an impregnating mixture containing paraffin, which increased the weight of the uniform by $40^{\circ}$ per cent, increased its water repellency and reduced its permeability to air, water, and water vapor. The uniforms were dry at the start of each day. The clothing was always worn with cotton shorts, socks and shoes. The trouser legs were tucked inside the socks, the jacket of shirt inside the trousers, and the clothing always completely buttoned.

The subjects entered the hot room to be weighed nude and to dress. After an initial 8 minutes the skin temperatures, pulse rate (standing erect) and rectal temperature were taken. The start of the march immediately followed. Pace discipline was enforced. The heart rate (marking time) and rectal temperature were taken again at the end of each hour, during a three minute break. At the end of the four hour march, or at the time of falling out, skin temperatures, pulse rate and rectal temperature were again taken, as well as a final nude weight. Water consumption $\left(0.1\right.$ per cent saline, $95^{\circ}$ F.) and urine output were recorded. Clothing was weighed before and immediately after each wearing. Skin temperatures of the cheek, chest, palm, forearm, and calf were determined radiometrically on the erect subject, the clothing being pushed away just sufficiently to permit placing the radiometer. The temperatures were integrated into an average skin temperature by the following weighting formula based on that of Hardy and Dubois (9) : cheek 0.10 , chest 0.44 , forearm 0.14 , calf 0.23 , palm 0.09 . Only the average weighted skin temperature is reported. Rectal temperatures were measured with calibrated clinical thermometers, pulse rates by palpation. Records were kept of the symptoms, complaints, general appearance, vigor and alertness of the men. Men were removed from test only when objective or subjective signs indicated it to be necessary.

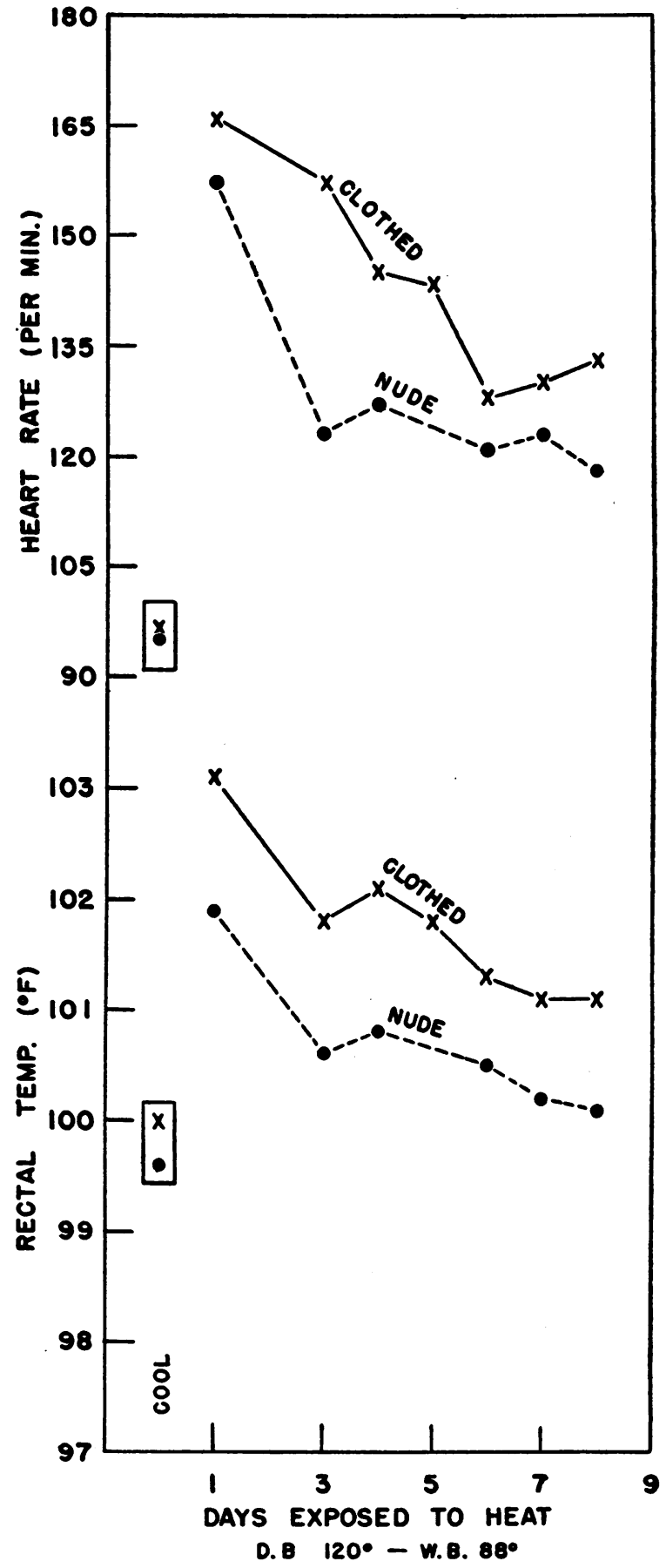

Fig. 1. Effect of Wearing Herringbone Twill Uniform on the Rate of Acclimatization

Data presented were obtained at the end of 1 hour march at D.B. $120^{\circ}-$ W.B. $88^{\circ} \mathrm{F}$. except that the data in blocks were taken on men working at D.B. $77^{\circ}-$ W.B. $62^{\circ} \mathrm{F}$. 
TABLE II

Physiologic effects of wearing standard herringbone twill clothing on acclimatized men working in the heat D.B. $120^{\circ} \mathrm{F}-$ W.B. $90^{\circ} \mathrm{F}$

\begin{tabular}{|c|c|c|c|c|c|c|c|c|c|c|c|c|c|c|}
\hline \multirow{2}{*}{ Name } & \multirow{2}{*}{ Clothing } & \multicolumn{5}{|c|}{ Rectal temperature ${ }^{\circ} \mathrm{F}$} & \multicolumn{5}{|c|}{ Pulse rate per min. } & \multirow{2}{*}{\multicolumn{2}{|c|}{$\frac{\begin{array}{l}\text { Skin temp. } \\
\text { (Avg. wtd.) }\end{array}}{0_{{ }_{\mathbf{F}}}^{\text {hours }} 4}$}} & \multirow{2}{*}{\begin{tabular}{|c|}
$\begin{array}{c}\text { Weight loss* } \\
\text { (Sweat) }\end{array}$ \\
$\begin{array}{c}\text { grams per } \\
\text { hour }\end{array}$
\end{tabular}} \\
\hline & & 0 & 1 & $\begin{array}{c}\text { hours } \\
2\end{array}$ & 3 & 4 & $\mathbf{0}$ & 1 & hours & 3 & 4 & & & \\
\hline $\mathrm{Hi}$ & $\begin{array}{l}\text { Nude } \\
\text { Clothed }\end{array}$ & $\begin{array}{l}98.7 \\
99.3\end{array}$ & $\begin{array}{r}99.7 \\
100.6\end{array}$ & $\begin{array}{l}100.0 \\
101.0\end{array}$ & $\begin{array}{r}99.9 \\
100.1\end{array}$ & $\begin{array}{r}99.9 \\
100.0\end{array}$ & $\begin{array}{l}108 \\
102\end{array}$ & $\begin{array}{l}123 \\
117\end{array}$ & $\begin{array}{l}111 \\
126\end{array}$ & $\begin{array}{l}111 \\
123\end{array}$ & $\begin{array}{l}117 \\
117\end{array}$ & $\begin{array}{l}96.5 \\
97.1\end{array}$ & $\begin{array}{l}96.4 \\
97.3\end{array}$ & $\begin{array}{l}1477 \\
1859\end{array}$ \\
\hline $\mathbf{K a}$ & $\begin{array}{l}\text { Nude } \\
\text { Clothed }\end{array}$ & $\begin{array}{l}98.6 \\
98.4\end{array}$ & $\begin{array}{r}99.7 \\
100.4\end{array}$ & $\begin{array}{r}99.8 \\
100.7\end{array}$ & $\begin{array}{r}99.7 \\
100.3\end{array}$ & $\begin{array}{r}99.8 \\
100.0\end{array}$ & $\begin{array}{l}102 \\
111\end{array}$ & $\begin{array}{l}123 \\
135\end{array}$ & $\begin{array}{l}123 \\
129\end{array}$ & $\begin{array}{l}120 \\
123\end{array}$ & $\begin{array}{l}114 \\
132\end{array}$ & $\begin{array}{l}96.3 \\
97.3\end{array}$ & $\begin{array}{l}97.2 \\
97.2\end{array}$ & $\begin{array}{l}1421 \\
1711\end{array}$ \\
\hline Lo & $\begin{array}{l}\text { Nude } \\
\text { Clothed }\end{array}$ & $\begin{array}{l}98.4 \\
98.4\end{array}$ & $\begin{array}{r}99.6 \\
100.7\end{array}$ & $\begin{array}{l}100.2 \\
101.2\end{array}$ & $\begin{array}{r}99.9 \\
100.9\end{array}$ & $\begin{array}{l}100.2 \\
100.8\end{array}$ & $\begin{array}{l}96 \\
96\end{array}$ & $\begin{array}{l}114 \\
120\end{array}$ & $\begin{array}{l}120 \\
129\end{array}$ & $\begin{array}{l}120 \\
132\end{array}$ & $\begin{array}{l}117 \\
135\end{array}$ & $\begin{array}{l}96.5 \\
97.3\end{array}$ & $\begin{array}{l}97.8 \\
97.9\end{array}$ & $\begin{array}{l}1645 \\
1543\end{array}$ \\
\hline $\mathbf{M i}$ & $\begin{array}{l}\text { Nude } \\
\text { Clothed }\end{array}$ & $\begin{array}{l}98.5 \\
98.3\end{array}$ & $\begin{array}{l}100.0 \\
100.7\end{array}$ & $\begin{array}{l}100.5 \\
101.2\end{array}$ & $\begin{array}{l}100.4 \\
101.0\end{array}$ & $\begin{array}{l}100.3 \\
101.0\end{array}$ & $\begin{array}{l}117 \\
102\end{array}$ & $\begin{array}{l}126 \\
120\end{array}$ & $\begin{array}{l}129 \\
153\end{array}$ & $\begin{array}{l}123 \\
138\end{array}$ & $\begin{array}{l}123 \\
138\end{array}$ & $\begin{array}{l}97.6 \\
97.3\end{array}$ & $\begin{array}{l}96.8 \\
97.3\end{array}$ & $\begin{array}{l}1901 \\
2123\end{array}$ \\
\hline Sc & $\begin{array}{l}\text { Nude } \\
\text { Clothed }\end{array}$ & $\begin{array}{l}99.0 \\
98.7\end{array}$ & $\begin{array}{l}100.4 \\
100.8\end{array}$ & $\begin{array}{l}100.3 \\
100.6\end{array}$ & $\begin{array}{r}99.9 \\
100.3\end{array}$ & $\begin{array}{l}100.1 \\
100.2\end{array}$ & $\begin{array}{l}111 \\
105\end{array}$ & $\begin{array}{l}129 \\
123\end{array}$ & $\begin{array}{l}126 \\
135\end{array}$ & $\begin{array}{l}123 \\
126\end{array}$ & $\begin{array}{l}123 \\
120\end{array}$ & $\begin{array}{l}97.7 \\
98.2\end{array}$ & $\begin{array}{l}96.9 \\
97.1\end{array}$ & $\begin{array}{l}2239 \\
2693\end{array}$ \\
\hline Mean & $\begin{array}{l}\text { Nude } \\
\text { Clothed }\end{array}$ & $\begin{array}{l}98.6 \\
98.6\end{array}$ & $\begin{array}{r}99.9 \\
100.6\end{array}$ & $\begin{array}{l}100.2 \\
100.9\end{array}$ & $\begin{array}{l}100.0 \\
100.5\end{array}$ & $\begin{array}{l}100.1 \\
100.4\end{array}$ & $\begin{array}{l}107 \\
103\end{array}$ & $\begin{array}{l}123 \\
123\end{array}$ & $\begin{array}{l}122 \\
134\end{array}$ & $\begin{array}{l}119 \\
128\end{array}$ & $\begin{array}{l}119 \\
128\end{array}$ & $\begin{array}{l}96.9 \\
97.4\end{array}$ & $\begin{array}{l}97.0 \\
97.4\end{array}$ & $\begin{array}{l}1737 \\
1986\end{array}$ \\
\hline
\end{tabular}

* The term weight loss is used to indicate gross sweat loss only. It does not represent an actual change in body weight since throughout all tests the men maintained body weights constant to within $\pm 0.5 \mathrm{kgm}$. by water replacement.

\section{RESULTS}

During the initial period of the three weeks' acclimatization to D.B. $120^{\circ}$-W.B. $88^{\circ}$ F., the ten men were divided into two groups to study the effect of clothing on acclimatization. One group wore herringbone twill uniforms each day and the other worked nude. During the first eight days the work load which started with one hour was increased by an hour every third day until the standard work period of four hours was reached. Only the man's rectal temperatures and pulse rates at the end of the first hour were stud-

TABLE III

Physiologic effects of wearing standard herringbone twill clothing on acclimatized men working in the heat D.B. $120^{\circ} \mathrm{F}-$ W.B. $92^{\circ} \mathrm{F}$

\begin{tabular}{|c|c|c|c|c|c|c|c|c|c|c|c|c|c|c|}
\hline \multirow{2}{*}{ Name } & \multirow{2}{*}{ Clothing } & \multicolumn{5}{|c|}{ Rectal temperature ${ }^{\circ} \mathbf{F}$} & \multicolumn{5}{|c|}{ Pulse rate per min. } & \multicolumn{2}{|c|}{$\begin{array}{l}\text { Skin temp. } \\
\text { (Avg. }{ }_{\text {otd }} \text {. }\end{array}$} & \multirow{2}{*}{$\begin{array}{c}\text { Weight loss } \\
\text { (Sweat) }\end{array}$} \\
\hline & & $\mathbf{0}$ & 1 & $\frac{\text { hours }}{2}$ & 3 & 4 & $\mathbf{0}$ & 1 & $\begin{array}{c}\text { hours } \\
2\end{array}$ & 3 & 4 & 0 & 4 & \\
\hline $\mathrm{Hi}$ & $\begin{array}{l}\text { Nude } \\
\text { Clothed }\end{array}$ & $\begin{array}{l}98.4 \\
98.2\end{array}$ & $\begin{array}{l}100.4 \\
100.8\end{array}$ & $\begin{array}{l}100.4 \\
101.7\end{array}$ & $\begin{array}{l}100.4 \\
101.7\end{array}$ & $\begin{array}{l}100.6 \\
102.0\end{array}$ & $\begin{array}{r}87 \\
102\end{array}$ & $\begin{array}{l}111 \\
129\end{array}$ & $\begin{array}{l}102 \\
111\end{array}$ & $\begin{array}{l}117 \\
147\end{array}$ & $\begin{array}{l}111 \\
123\end{array}$ & $\begin{array}{l}97.2 \\
97.9\end{array}$ & $\begin{array}{l}98.7 \\
99.4\end{array}$ & $\begin{array}{l}1562 \\
1688\end{array}$ \\
\hline $\mathbf{K a}$ & $\begin{array}{l}\text { Nude } \\
\text { Clothed }\end{array}$ & $\begin{array}{l}98.6 \\
98.7\end{array}$ & $\begin{array}{l}100.0 \\
101.2\end{array}$ & $\begin{array}{l}100.0 \\
102.0\end{array}$ & $\begin{array}{l}100.2 \\
102.0\end{array}$ & $\begin{array}{l}100.7 \\
101.8\end{array}$ & $\begin{array}{r}96 \\
105\end{array}$ & $\begin{array}{l}120 \\
150\end{array}$ & $\begin{array}{l}120 \\
147\end{array}$ & $\begin{array}{l}132 \\
141\end{array}$ & $\begin{array}{l}123 \\
150\end{array}$ & $\begin{array}{l}97.1 \\
97.2\end{array}$ & $\begin{array}{l}98.5 \\
99.3\end{array}$ & $\begin{array}{l}1684 \\
1774\end{array}$ \\
\hline Lo & $\begin{array}{l}\text { Nude } \\
\text { Clothed }\end{array}$ & $\begin{array}{l}98.6 \\
98.9\end{array}$ & $\begin{array}{l}100.1 \\
102.0\end{array}$ & $\begin{array}{l}101.4 \\
103.1\end{array}$ & 101.7 & 102.1 & $\begin{array}{l}102 \\
105\end{array}$ & $\begin{array}{l}144 \\
144\end{array}$ & $\begin{array}{l}129 \\
144\end{array}$ & 120 & 132 & $\begin{array}{l}98.1 \\
98.0\end{array}$ & $\begin{array}{c}99.4 \\
*\end{array}$ & ${ }_{*}^{1396}$ \\
\hline Mi & $\begin{array}{l}\text { Nude } \\
\text { Clothed }\end{array}$ & $\begin{array}{l}98.8 \\
98.8\end{array}$ & $\begin{array}{l}100.3 \\
101.3\end{array}$ & $\begin{array}{l}100.4 \\
101.8\end{array}$ & $\underset{t}{100.5}$ & 101.0 & $\begin{array}{l}105 \\
129\end{array}$ & $\begin{array}{l}126 \\
147\end{array}$ & $\begin{array}{l}150 \\
141\end{array}$ & $\begin{array}{c}123 \\
t\end{array}$ & 126 & $\begin{array}{l}97.8 \\
98.3\end{array}$ & $\stackrel{98.7}{t}$ & $\stackrel{2071}{t}$ \\
\hline Sc & $\begin{array}{l}\text { Nude } \\
\text { Clothed }\end{array}$ & $\begin{array}{l}99.3 \\
99.0\end{array}$ & $\begin{array}{l}101.4 \\
102.0\end{array}$ & $\begin{array}{l}101.2 \\
102.3\end{array}$ & $\begin{array}{l}101.2 \\
102.4\end{array}$ & $\begin{array}{l}101.5 \\
102.5\end{array}$ & $\begin{array}{l}111 \\
114\end{array}$ & $\begin{array}{l}156 \\
147\end{array}$ & $\begin{array}{l}123 \\
132\end{array}$ & $\begin{array}{l}120 \\
132\end{array}$ & $\begin{array}{l}126 \\
126\end{array}$ & $\begin{array}{l}97.8 \\
98.1\end{array}$ & $\begin{array}{l}98.2 \\
99.2\end{array}$ & $\begin{array}{l}2543 \\
2477\end{array}$ \\
\hline Mean & $\begin{array}{l}\text { Nude } \\
\text { Clothed }\end{array}$ & $\begin{array}{l}98.7 \\
98.7\end{array}$ & $\begin{array}{l}100.4 \\
101.5\end{array}$ & $\begin{array}{l}100.7 \\
102.2\end{array}$ & 100.8 & 101.2 & $\begin{array}{l}100 \\
111\end{array}$ & $\begin{array}{l}131 \\
143\end{array}$ & $\begin{array}{l}125 \\
135\end{array}$ & 122 & 124 & $\begin{array}{l}97.6 \\
97.9\end{array}$ & 8.7 & 1851 \\
\hline
\end{tabular}

* Unable to continue after 2.4 hours-final data: $103.2,150,100.4$ and 1589.

† Unable to continue after 2.7 hours-final data: 102.4, 156, 99.6 and 2063. 
TABLE IV

Physiologic effects of wearing standard herringbone twill clothing on acclimatized men working in the heat D.B. $93^{\circ} \mathrm{F}-$ W.B. $92^{\circ} \mathrm{F}$

\begin{tabular}{|c|c|c|c|c|c|c|c|c|c|c|c|c|c|c|}
\hline \multirow{2}{*}{ Name } & \multirow{2}{*}{ Clothing } & \multicolumn{5}{|c|}{ Rectal temperature ${ }^{\circ} \mathrm{F}$} & \multicolumn{5}{|c|}{ Pulse rate per min. } & \multirow{2}{*}{\multicolumn{2}{|c|}{$\frac{\underbrace{}_{\text {Skin temp. }}}{\text { (Avg. }_{\text {Ftd.) }}}$}} & \multirow{2}{*}{$\frac{\begin{array}{c}\text { Weight loss } \\
\text { (Sweat) }\end{array}}{\underbrace{\text { hour }}_{\text {grams per }}}$} \\
\hline & & 0 & 1 & hours & 3 & 4 & $\mathbf{0}$ & 1 & hours & 3 & 4 & & & \\
\hline Di & $\begin{array}{l}\text { Nude } \\
\text { Clothed }\end{array}$ & $\begin{array}{l}98.8 \\
98.5\end{array}$ & $\begin{array}{l}100.0 \\
101.0\end{array}$ & $\begin{array}{l}100.4 \\
101.1\end{array}$ & $\begin{array}{l}100.6 \\
101.3\end{array}$ & $\begin{array}{l}100.6 \\
101.4\end{array}$ & $\begin{array}{l}93 \\
93\end{array}$ & $\begin{array}{l}129 \\
135\end{array}$ & $\begin{array}{l}114 \\
135\end{array}$ & $\begin{array}{l}123 \\
123\end{array}$ & $\begin{array}{l}117 \\
132\end{array}$ & $\begin{array}{l}95.3 \\
95.6\end{array}$ & $\begin{array}{l}96.4 \\
97.8\end{array}$ & $\begin{array}{l}1706 \\
1760\end{array}$ \\
\hline $\mathbf{K n}$ & $\begin{array}{l}\text { Nude } \\
\text { Clothed }\end{array}$ & $\begin{array}{l}98.4 \\
98.5\end{array}$ & $\begin{array}{r}99.8 \\
100.9\end{array}$ & $\begin{array}{l}100.1 \\
101.2\end{array}$ & $\begin{array}{l}100.4 \\
101.0\end{array}$ & $\begin{array}{l}100.3 \\
101.0\end{array}$ & $\begin{array}{r}90 \\
108\end{array}$ & $\begin{array}{l}114 \\
120\end{array}$ & $\begin{array}{l}117 \\
129\end{array}$ & $\begin{array}{l}117 \\
129\end{array}$ & $\begin{array}{l}120 \\
123\end{array}$ & $\begin{array}{l}94.8 \\
95.8\end{array}$ & $\begin{array}{l}97.0 \\
98.2\end{array}$ & $\begin{array}{l}1848 \\
1824\end{array}$ \\
\hline $\mathbf{L i}$ & $\begin{array}{l}\text { Nude } \\
\text { Clothed }\end{array}$ & $\begin{array}{l}99.7 \\
99.4\end{array}$ & $\begin{array}{l}100.7 \\
101.7\end{array}$ & $\begin{array}{l}100.6 \\
102.3\end{array}$ & $\begin{array}{l}100.7 \\
102.0\end{array}$ & $\begin{array}{l}100.6 \\
102.1\end{array}$ & $\begin{array}{r}93 \\
105\end{array}$ & $\begin{array}{l}117 \\
135\end{array}$ & $\begin{array}{l}108 \\
126\end{array}$ & $\begin{array}{l}117 \\
144\end{array}$ & $\begin{array}{l}126 \\
144\end{array}$ & $\begin{array}{l}96.4 \\
96.6\end{array}$ & $\begin{array}{l}96.5 \\
98.1\end{array}$ & $\begin{array}{l}1908 \\
2353\end{array}$ \\
\hline $\mathbf{M a}$ & $\begin{array}{l}\text { Nude } \\
\text { Clothed }\end{array}$ & $\begin{array}{l}98.4 \\
98.1\end{array}$ & $\begin{array}{l}100.3 \\
100.9\end{array}$ & $\begin{array}{l}100.7 \\
101.6\end{array}$ & $\begin{array}{l}100.8 \\
101.3\end{array}$ & $\begin{array}{l}100.6 \\
101.6\end{array}$ & $\begin{array}{l}120 \\
102\end{array}$ & $\begin{array}{l}150 \\
141\end{array}$ & $\begin{array}{l}153 \\
144\end{array}$ & $\begin{array}{l}141 \\
150\end{array}$ & $\begin{array}{l}138 \\
156\end{array}$ & $\begin{array}{l}95.3 \\
95.9\end{array}$ & $\begin{array}{l}96.7 \\
97.6\end{array}$ & $\begin{array}{l}1791 \\
2244\end{array}$ \\
\hline $\mathrm{Sz}$ & $\begin{array}{l}\text { Nude } \\
\text { Clothed }\end{array}$ & $\begin{array}{l}98.0 \\
98.7\end{array}$ & $\begin{array}{r}99.4 \\
100.6\end{array}$ & $\begin{array}{r}99.5 \\
101.5\end{array}$ & $\begin{array}{r}99.8 \\
101.0\end{array}$ & $\begin{array}{r}99.8 \\
100.7\end{array}$ & $\begin{array}{r}87 \\
105\end{array}$ & $\begin{array}{l}108 \\
129\end{array}$ & $\begin{array}{l}120 \\
141\end{array}$ & $\begin{array}{l}123 \\
132\end{array}$ & $\begin{array}{l}123 \\
123\end{array}$ & $\begin{array}{l}95.2 \\
95.9\end{array}$ & $\begin{array}{l}96.8 \\
97.9\end{array}$ & $\begin{array}{l}1705 \\
1760\end{array}$ \\
\hline Mean & $\begin{array}{l}\text { Nude } \\
\text { Clothed }\end{array}$ & $\begin{array}{l}98.7 \\
98.6\end{array}$ & $\begin{array}{l}100.0 \\
101.0\end{array}$ & $\begin{array}{l}100.3 \\
101.5\end{array}$ & $\begin{array}{l}100.5 \\
101.3\end{array}$ & $\begin{array}{l}100.4 \\
101.4\end{array}$ & $\begin{array}{r}97 \\
103\end{array}$ & $\begin{array}{l}124 \\
132\end{array}$ & $\begin{array}{l}122 \\
135\end{array}$ & $\begin{array}{l}124 \\
136\end{array}$ & $\begin{array}{l}125 \\
136\end{array}$ & $\begin{array}{l}95.4 \\
96.0\end{array}$ & $\begin{array}{l}96.7 \\
97.9\end{array}$ & $\begin{array}{l}1792 \\
1988\end{array}$ \\
\hline
\end{tabular}

ied. The first hour was chosen for presentation the unacclimatized individual than in the acclimasince it was the only work load accomplished by tized. the entire group on all days. The difference in pulse rate and rectal temperature remained constant between the two groups in response to this constant work stimulus (Figure 1). The relative effect of the clothing appeared to be no greater in

\section{UPPER LIMIT STUDIES}

Data on the upper limits for the subjects working both nude and clothed in ordinary herringbone twill are presented in Tables II to V, and Figures

TABLE $\mathbf{V}$

Physiologic effects of wearing standard herringbone twill clothing on acclimatized men working in the heat D.B. $95^{\circ} \mathrm{F}-$ W.B. $94^{\circ} \mathrm{F}$

\begin{tabular}{|c|c|c|c|c|c|c|c|c|c|c|c|c|c|c|}
\hline \multirow{2}{*}{ Name } & \multirow{2}{*}{ Clothing } & \multicolumn{5}{|c|}{ Rectal temperature ${ }^{\circ} \mathbf{F}$} & \multicolumn{5}{|c|}{ Pulse rate per min. } & \multicolumn{2}{|c|}{$\begin{array}{l}\text { Skin temp. } \\
\text { (Avg. wtd.) }\end{array}$} & \multirow{2}{*}{$\frac{\begin{array}{c}\text { Weight loss } \\
\text { (Sweat) }\end{array}}{\underbrace{\text { hour }}_{\text {grams per }}}$} \\
\hline & & $\mathbf{0}$ & 1 & hours & 3 & 4 & $\mathbf{0}$ & 1 & hours & 3 & 4 & $0^{n}$ & $s_{4}$ & \\
\hline $\mathbf{H i}$ & $\begin{array}{l}\text { Nude } \\
\text { Clothed }\end{array}$ & $\begin{array}{l}98.7 \\
99.0\end{array}$ & $\begin{array}{l}100.4 \\
101.7\end{array}$ & $\begin{array}{l}101.3 \\
102.8\end{array}$ & 101.1 & 101.1 & $\begin{array}{r}96 \\
114\end{array}$ & $\begin{array}{l}114 \\
144\end{array}$ & $\begin{array}{l}132 \\
135\end{array}$ & 132 & 123 & $\begin{array}{l}95.4 \\
96.6\end{array}$ & $\begin{array}{c}96.8 \\
*\end{array}$ & 1806 \\
\hline $\mathbf{K a}$ & $\begin{array}{l}\text { Nude } \\
\text { Clothed }\end{array}$ & $\begin{array}{l}98.4 \\
98.6\end{array}$ & $\begin{array}{l}100.0 \\
101.3\end{array}$ & $\begin{array}{l}100.8 \\
102.7\end{array}$ & $\stackrel{100.7}{\dagger}$ & 101.0 & $\begin{array}{l}114 \\
114\end{array}$ & $\begin{array}{l}141 \\
159\end{array}$ & $\begin{array}{l}147 \\
171\end{array}$ & 135 & 156 & $\begin{array}{l}95.6 \\
96.1\end{array}$ & $\stackrel{97.2}{t}$ & $\begin{array}{c}1600 \\
t\end{array}$ \\
\hline Lo & $\begin{array}{l}\text { Nude } \\
\text { Clothed }\end{array}$ & $\begin{array}{l}99.1 \\
99.1\end{array}$ & $\begin{array}{l}100.8 \\
102.2\end{array}$ & $\begin{array}{l}101.6 \\
103.5\end{array}$ & 102.2 & 102.6 & $\begin{array}{l}108 \\
114\end{array}$ & $\begin{array}{l}132 \\
156\end{array}$ & $\begin{array}{l}135 \\
156\end{array}$ & 138 & 141 & $\begin{array}{l}96.4 \\
96.7\end{array}$ & $\stackrel{98.1}{\ddagger}$ & $\begin{array}{c}1771 \\
\ddagger\end{array}$ \\
\hline $\mathbf{M i}$ & $\begin{array}{l}\text { Nude } \\
\text { Clothed }\end{array}$ & $\begin{array}{r}100.0 \\
98.6\end{array}$ & $\begin{array}{l}100.1 \\
101.6\end{array}$ & $\begin{array}{c}101.0 \\
8\end{array}$ & 101.0 & 101.4 & $\begin{array}{l}102 \\
120\end{array}$ & $\begin{array}{l}129 \\
162\end{array}$ & $\begin{array}{c}132 \\
8\end{array}$ & 138 & 144 & $\begin{array}{l}95.5 \\
96.4\end{array}$ & $\begin{array}{c}97.4 \\
8\end{array}$ & 2500 \\
\hline Sc & $\begin{array}{l}\text { Nude } \\
\text { Clothed }\end{array}$ & $\begin{array}{l}99.1 \\
98.8\end{array}$ & $\begin{array}{l}100.4 \\
101.7\end{array}$ & $\begin{array}{l}101.1 \\
102.6\end{array}$ & $\begin{array}{l}101.7 \\
103.0\end{array}$ & $\begin{array}{l}101.5 \\
102.9\end{array}$ & $\begin{array}{l}105 \\
108\end{array}$ & $\begin{array}{l}138 \\
150\end{array}$ & $\begin{array}{l}144 \\
153\end{array}$ & $\begin{array}{l}138 \\
150\end{array}$ & $\begin{array}{l}132 \\
156\end{array}$ & $\begin{array}{l}94.7 \\
95.9\end{array}$ & $\begin{array}{l}97.2 \\
99.2\end{array}$ & $\begin{array}{l}3087 \\
2586\end{array}$ \\
\hline Mean & $\begin{array}{l}\text { Nude } \\
\text { Clothed }\end{array}$ & $\begin{array}{l}99.1 \\
98.8\end{array}$ & $\begin{array}{l}100.3 \\
101.7\end{array}$ & 101.2 & 101.3 & 101.5 & $\begin{array}{l}105 \\
114\end{array}$ & $\begin{array}{l}131 \\
154\end{array}$ & 138 & 136 & 139 & $\begin{array}{l}95.5 \\
96.3\end{array}$ & 97.3 & 2153 \\
\hline
\end{tabular}

* Unable to continue after 2.2 hours-final data: 102.7, 150, 98.4 and 1804.

+ Unable to continue after 2.0 hours-final data: 102.7, 171, 98.3 and 2243 .

‡ Unable to continue after 2.8 hours-final data: 103.4, 150, 99.1 and 1626 .

Unable to continue after 1.8 hours-final data: 102.9, 144, 98.3 and 2356 . 


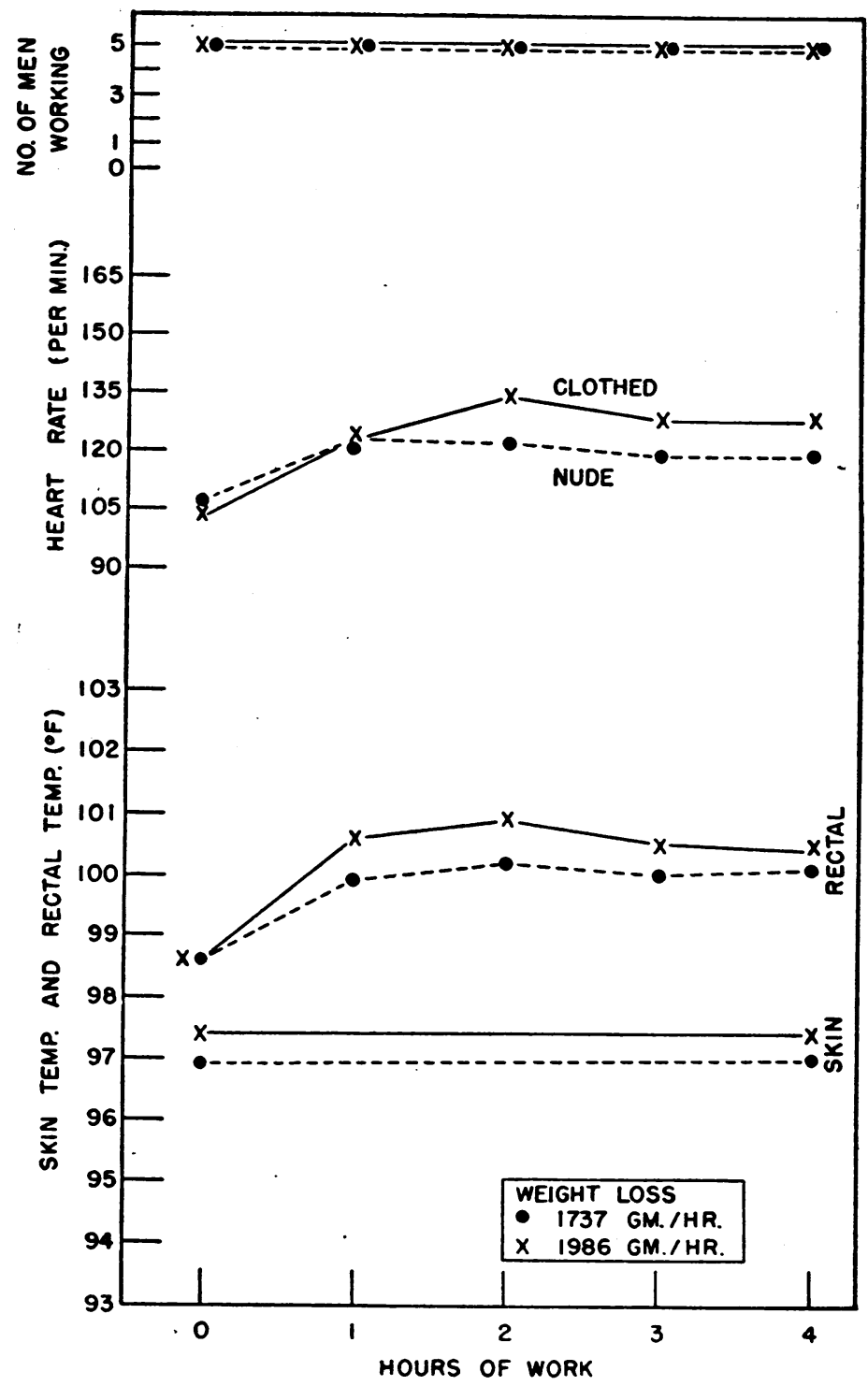

D.B. $120^{\circ}$ - W. B. $90^{\circ}$

Fig. 2. Average Physiologic Effects of Wearing Standard Herringbone Twill Uniforms on Acclimatized Men MarchING 3 мPH. AT D.B. $120^{\circ}-$ W.B. $90^{\circ}$ F.

This is one of the upper limiting environments for four hours of work in the clothed man. Key, ..... nude; $\mathrm{x}-\mathrm{x}$ clothed.

2 to 6 . In view of the impossibility of elaborately characterizing the special impregnated garments at this time, data on the men wearing these have been omitted but all the pertinent conclusions will be presented in the text.

Environments with D.B. $120^{\circ} \mathrm{F}$

When nude, subjects were able to complete the required 4 hours of marching in environments with wet bulb temperatures up to and including $92^{\circ} \mathrm{F}$. However, when clothed in herringbone twill, they were able to complete, as a group, 4 hours of marching at a W.B. of $90^{\circ} \mathrm{F}$. but not $92^{\circ} \mathrm{F}$. At W.B. $92^{\circ} \mathrm{F}$., although three of the men could finish 4 hours two were unable to complete even 3 hours of work. When clothed in the special impregnated herringbone twill the men could not complete, as a group, four hours of marching at 


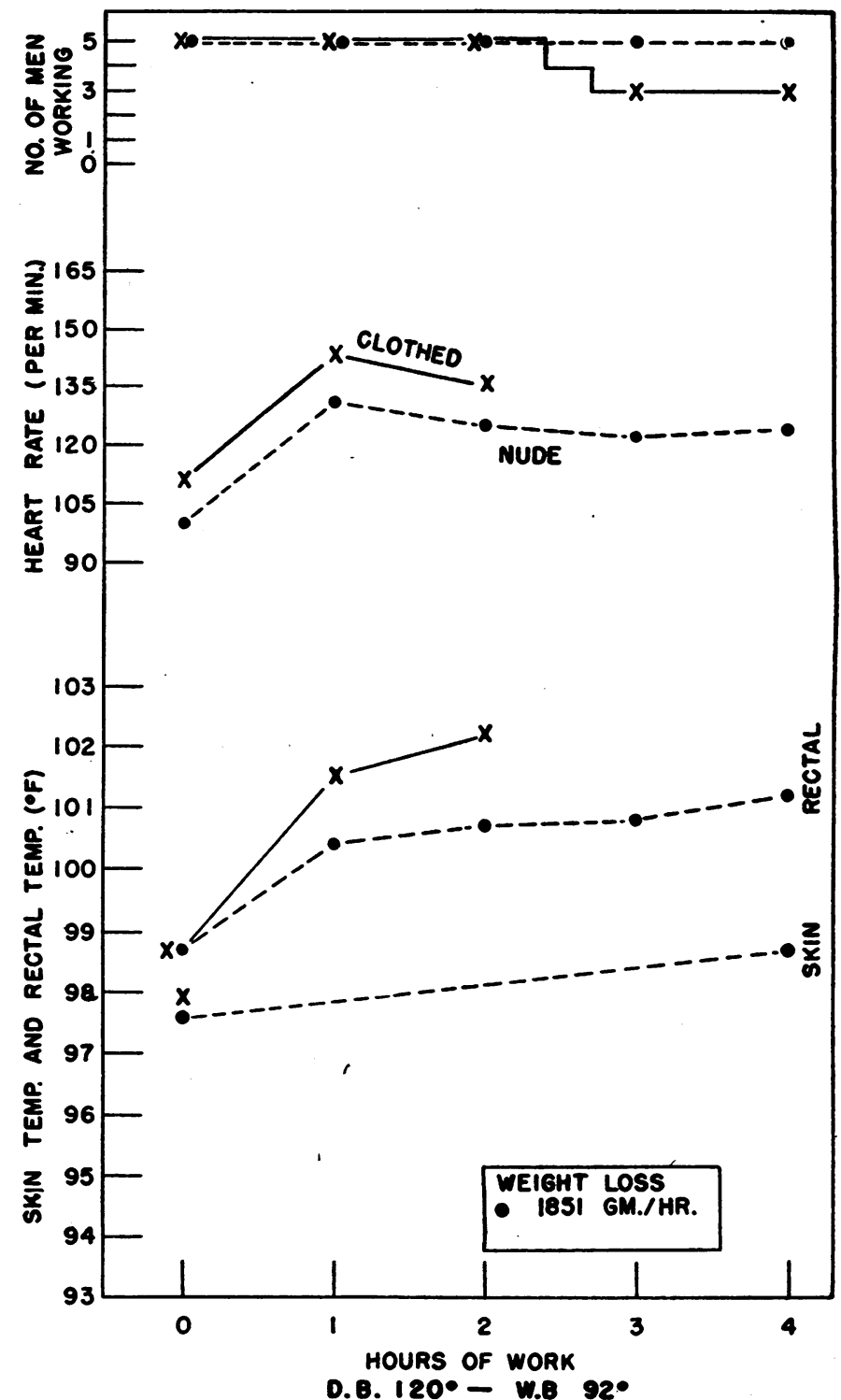

Fig. 3. Average Physiologic Effects of Wearing Standard Herringbone Twill Uniforms on Acchimatized Men MarchING 3 MPH. AT D.B. $120^{\circ}$-W.B. $92^{\circ}$ F.

This is above the upper limits for four hours of work for the group when clothed since two men failed to finish. Unless group finishes comparable period of work data not plotted. Key, -.-.. nude; $x-x$ clothed.

wet bulb temperatures above $88^{\circ} \mathrm{F}$. Hence it was found (Figure 6) that the upper limiting wet bulb temperature for successful group performance of 4 hours of marching at D.B. $120^{\circ} \mathrm{F}$., was $92^{\circ} \mathrm{F}$. for nude men, $90^{\circ} \mathrm{F}$. for herringbone twill clothed, and $88^{\circ} \mathrm{F}$. for men wearing the specially treated herringbone twill uniform.
Environments with D.B. $93^{\circ}$ and $95^{\circ} \mathrm{F}$.

At D.B. $95^{\circ} \mathrm{F}$., the group when nude was able to march 4 hours at wet bulb temperatures up to and including $94^{\circ} \mathrm{F}$. This demonstrates the importance of the wet bulb temperature in this zone of evaporative heat regulation, in critically limiting man's performance since a reduction of the dry 


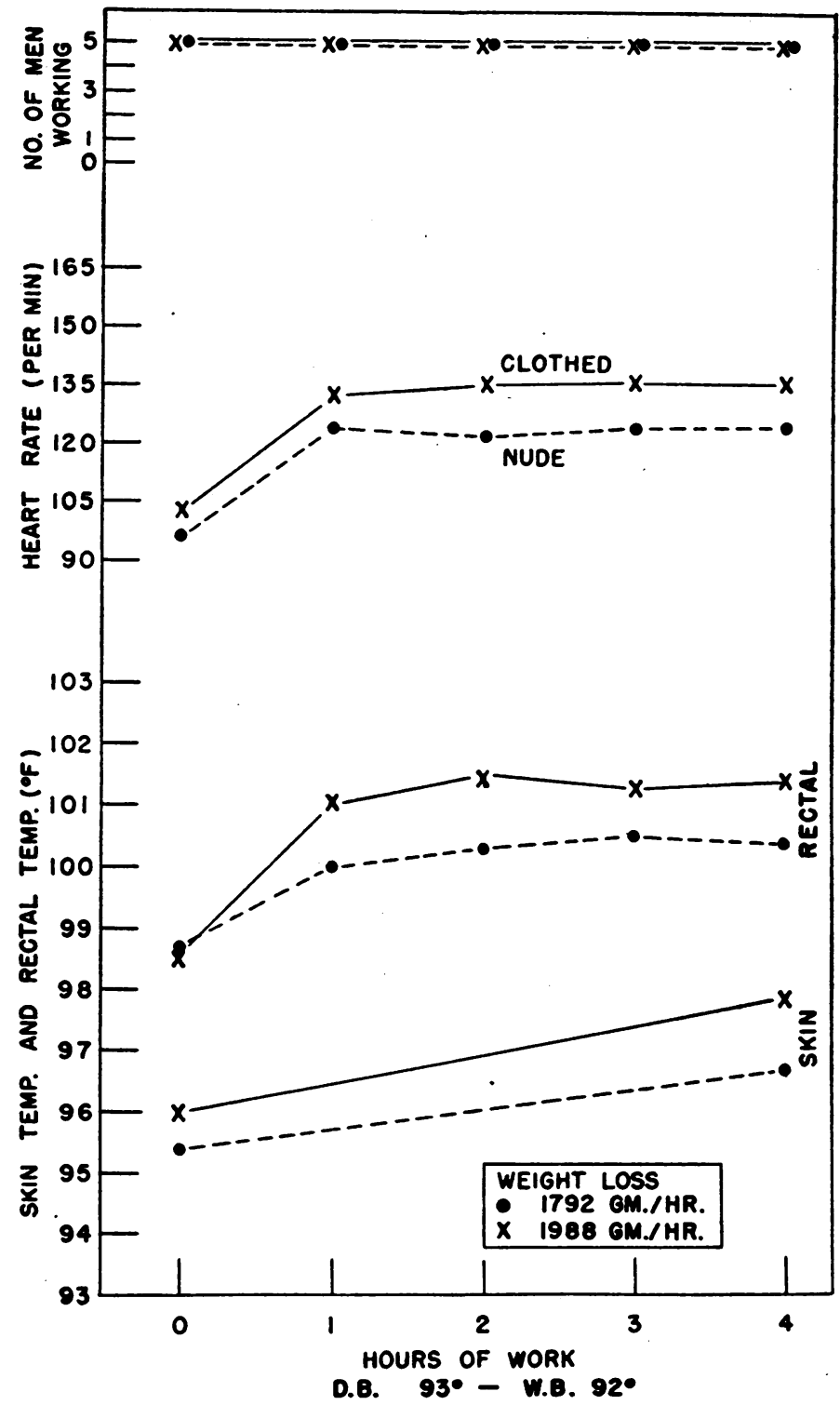

Fig. 4. Average Physiologic Effects of Wearing Standard Herringbone Twill Uniforms on Acclimatized Men MarchING 3 м.н. AT D.B. $93^{\circ}$-W.B. $92^{\circ}$ F.

This is one of the upper limiting environments for four hours of work in the clothed man. Key, ..... nude; $\mathbf{x}-\mathbf{x}$ clothed.

bulb temperature by $25^{\circ}$ resulted in elevating the wet bulb upper limit by only $2^{\circ} \mathrm{F}$. When the men worked in either the untreated or treated herringbone twill, the upper limit was D.B. $93^{\circ}-$ W.B. $92^{\circ} \mathrm{F}$. Thus, in the saturated environment, the two types of clothing could not be clearly distinguished on the basis of group performance.

\section{Physiological Responses}

The effect of clothing is not restricted to its effect on work performance alone, but it is reflected also in significant physiologic changes in the rectal and skin temperatures and pulse of men working in hot climates. These physiological changes (Tables II toV) should be contrasted with the minimal effect of clothing on these men performing the standard work in a relatively cool environment. The following data compare the group averages of the nude and clothed men at the 


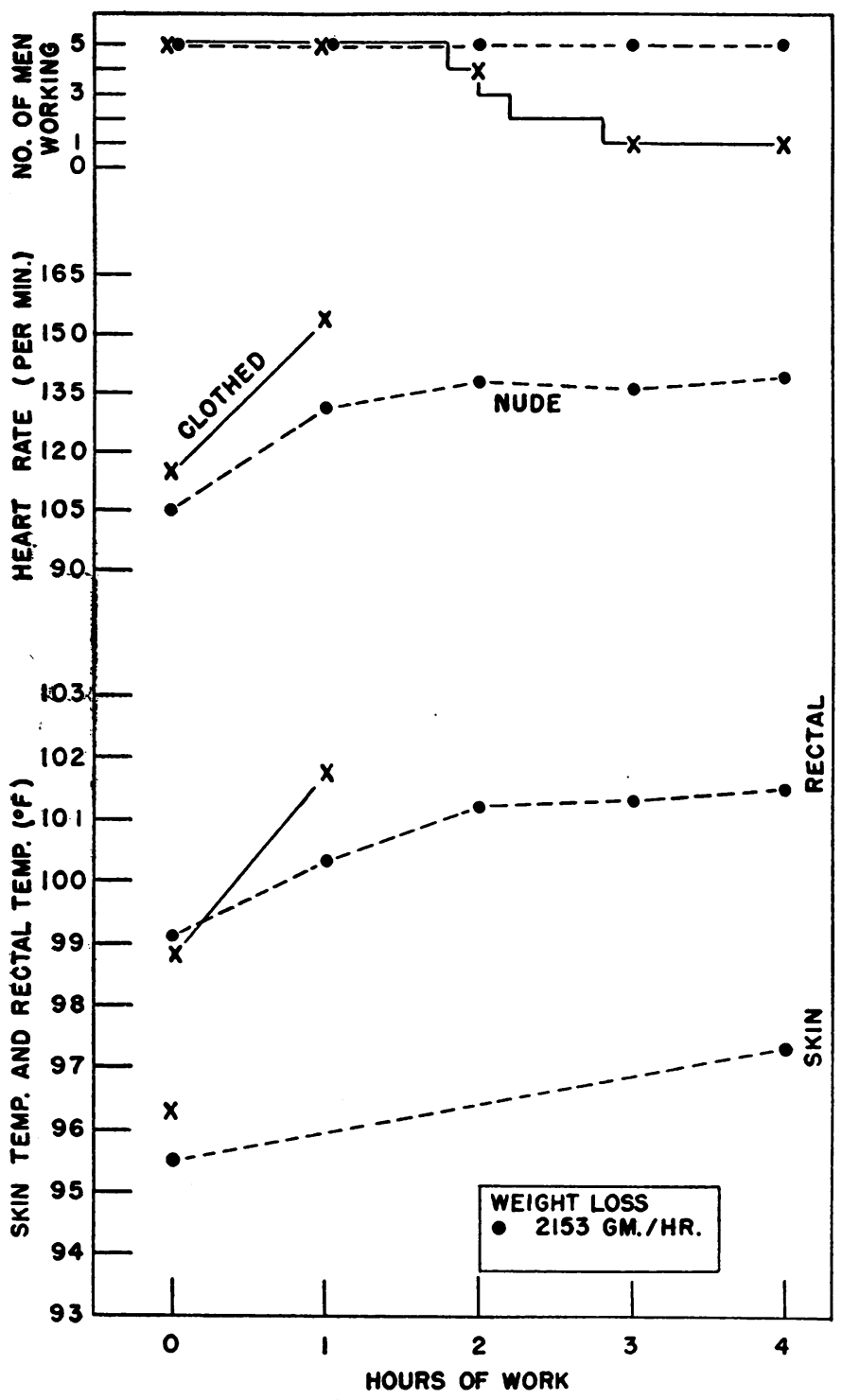

D.B. $95^{\circ}-$ W.B. $94^{\circ}$

Fig. 5. Average Physiologic Effects of Wearing Standard Herringbone Twill Uniforms on Acclimatized Men MarchING 3 мPH. AT D.B. $95^{\circ}-$ W.B. $93^{\circ}$ F.

This is above the upper limits for four hours of work for the group when clothed since four of the five men failed to finish. Unless group finishes comparable period of work data not plotted.

Key, .-... nude; $\mathbf{x} \longrightarrow \mathbf{x}$ clothed.

end of the four hours of marching at D.B. $77^{\circ}-$ W.B. $62^{\circ} \mathrm{F}$.

\begin{tabular}{l|c|c|c|c}
\hline \hline & $\begin{array}{c}\text { Rectal } \\
\text { temp. } \\
{ }_{\mathrm{F}} \mathbf{F}\end{array}$ & $\begin{array}{c}\text { Heart rate } \\
\text { per min. }\end{array}$ & $\begin{array}{c}\text { Skin temp. } \\
{ }^{\mathbf{F}}\end{array}$ & $\begin{array}{c}\text { Weight loss } \\
\text { grams per } \\
\text { hour }\end{array}$ \\
\hline $\begin{array}{l}\text { Nude } \\
\text { Clothed }\end{array}$ & 99.6 & 91 & 90.8 & 143 \\
\hline 9.8 & 106 & 91.3 & 281 \\
\hline
\end{tabular}

Clothing imposed a significant heat load in the hot environments studied. At the end of four hours in the two environments in which all men finished, whether nude or clothed (Tables II and IV) the rectal temperature of the clothed men ranged from $0.1^{\circ}$ to $1.5^{\circ} \mathrm{F}$. higher than the same man nude; the skin temperature of the clothed man from $0.0^{\circ}$ to $1.6^{\circ} \mathrm{F}$. higher than nude; the pulse 


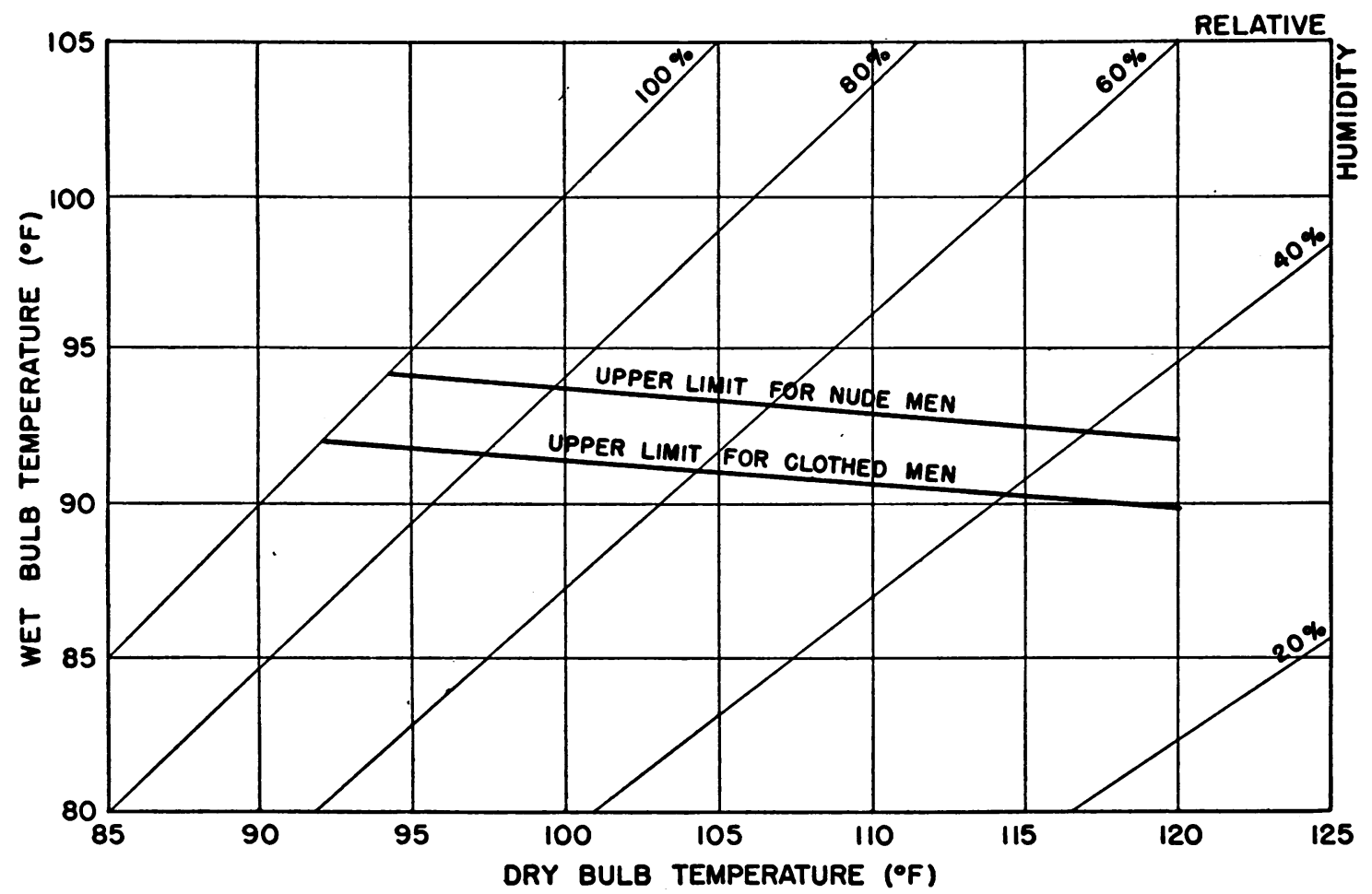

Fig. 6. Effect of Wearing Standard Herringbone Twill Uniforms on the Upper Limits of Heat Tolerated by Working Acclimatized Men

The group nude or clothed was unable to work at a rate of $250 \mathrm{Cal}$. per hour for four hours at levels of heat above those shown.

rate from 3 beats lower to 18 beats higher than the nude; the sweat rate from 24 grams lower to 454 grams higher. The wearing of the special impregnated clothing resulted in proportionately greater changes which ranged up to approximately twice as.great as those induced by wearing ordinary herringbone twill.

In addition to the physiologic cost, the men exhibited striking subjective reactions. These responses correlated well with the physiological findings. The men felt much cooler in the nude than when they wore either the untreated or treated clothing. In addition, the treated clothing was considered to be appreciably warmer than ordinary herringbone twill uniforms.

Weighing the clothing before and after each wearing revealed the herringbone twill clothing had absorbed approximately 1400 grams of sweat, the special clothing absorbed only half as much as this.

\section{DISCUSSION}

This study has described the acute physiological heat tolerance of a group of clothed acclimatized men exposed to several controlled external and internal heat loads. It confirms and complements observations made previously at this laboratory (6) on the upper limits tolerated by nude men. It must not be expected that industrial workers can work effectively at these levels. Rather do these upper limits indicate the maximal performance that is physiologically attainable by a group in severe heat. They may serve, therefore, as a rough guide of endurance of men in industrial operation in which optimal conditions cannot be achieved.

The specific type of work and environment, the acute nature of the experiment, the high state of acclimatization, motivation, and physical fitness of the subjects, all limit extensive direct application of this and other laboratory data. For example, the presence of a large source of radiant heat would considerably lower the limiting wet bulb temperature (10). As Von Schlictegroll (11) has stressed, ideally, specific practical studies should be carried out in the hot industries.

It is important to stress the great individual variability seen in the data. From a practical standpoint the reactions are analyzed in terms of 
the average group response. However, any individual's performance and reactions may depart significantly from that of the group.

In additional work on another problem (12) at this laboratory, twelve men clothed in herringbone twill were fully acclimatized to marching at D.B. $120^{\circ}$-W.B. $93^{\circ}$ and D.B. $120^{\circ}-$ W.B. $90^{\circ}$ F. It was found that D.B. $120^{\circ}-$ W.B. $93^{\circ} \mathrm{F}$. was above the upper limits while all the men were able to work for four hours (250 Cal. per hour) at D.B. $120^{\circ}-$ W.B. $90^{\circ} \mathrm{F}$. This finding confirmed one of the upper limits described in the present paper.

Robinson, Turrell and Gerking (8) approached the problem of limiting hot environments from the standpoint of thermal equilibrium while this laboratory has used different criteria; nevertheless, both agree as to the upper limits for the nude men. At equivalent work rate $\left(130 \mathrm{Cal}\right.$. per $\mathrm{M}^{2}$ per hour) Robinson found the limiting environments for nude men similar to those reported by this laboratory, i.e. D.B. $95^{\circ}$-W.B. $94^{\circ}$; D.B. $122.0^{\circ}-$ W.B. $92.0^{\circ} \mathrm{F}$. In clothed men the limits determined by Robinson and by this study are similar for the saturated environments, but not for the $120^{\circ} \mathrm{F}$. environments. Robinson's group, studying windbreak poplin, set the limit at about D.B. $122^{\circ}$ -W.B. $86^{\circ}$ F., while this study indicated the limit to be D.B. $120^{\circ}-$ W.B. $90^{\circ}$ F. for men clothed in herringbone twill and D.B. $120^{\circ}$-W.B. $88^{\circ}$ F. for men in impregnated herringbone twill.

The explanation for the difference at the $120^{\circ} \mathrm{F}$. environment is felt to be related to the type of clothing worn by the subjects. In these environments clothing plays a decisive rôle in determining upper limits, since evaporation and its cooling effect is limited by the type of clothing. On the other hand, in hot saturated environments evaporation of sweat is critically limited by the humidity, and the type of clothing plays a less important rôle.

\section{SUMMARY}

The effect of wearing two types of clothing on the ability of acclimatized men to work at the upper limits of heat has been evaluated in laboratory studies. The upper limiting wet bulb temperature for successful group performance of 4 hours of marching at $3 \mathrm{mph}$ (250 Cal. per hour) in an environment with D.B. $120^{\circ} \mathrm{F}$., was $92^{\circ} \mathrm{F}$. for nude men, $90^{\circ} \mathrm{F}$. for herringbone twill clothed and $88^{\circ} \mathrm{F}$. for men wearing an impregnated herringbone twill uniform. The upper limiting wet bulb temperature at a D.B. $93^{\circ}$ to $95^{\circ}$, was $94^{\circ} \mathrm{F}$. for nude men and $92^{\circ} \mathrm{F}$. for men clothed either in treated or untreated herringbone twill uniforms.

At the upper limits of environmental heat, the wearing of a single layer herringbone twill $(8 \mathrm{oz}$. uniform imposes a heat load equivalent to a $2^{\circ} \mathrm{F}$. increase in the wet bulb temperature.

The authors are indebted to $T / 3$ 's $K$. C. Davis, $H$. Golden, J. P. Stack, J. E. Wagar, A. F. Kunes, T/4 R. A. Kleinman, T/5 W. J. Robinson, and Mr. J. W. Gregg, for technical assistance in these experiments.

\section{BIBLIOGRAPHY}

1. Haldane, J. S., The influence of high air temperatures. J. Hyg., 1905, 5, 494.

2. Vernon, H. M., The kata-thermometer in studies of body heat and efficiency. Report 73, Med. Res. Council, 1923.

3. McConnell, W. J., Houghten, F. C., and Yagloglou, C. P., Air motion-high temperatures and various humidities-reactions on human beings. Trans. Am. Soc. Heat \& Vent. Eng., 1924, 30, 167.

4. Dreosti, A. O., Problems, arising out of temperature and humidity in deep mining on the Witwatersrand. J. Chem. Met. Soc. South Africa, 1935, 36, 102.

5. Caplan, A., A critical analysis of collapse in underground workers on the Kolar Gold Field. Bull. Inst. Min. \& Metall., 1943, 463, 1.

6. Eichna, L. W., Ashe, W. F., Bean, W. B., and Shelley, W. B., The upper limits of environmental heat and humidity tolerated by acclimatized men working in hot environments. J. Indust. Hyg. \& Toxicol., 1945, 27, 59.

7. Gagge, A. P., Winslow, C. E. A., and Herrington, L. P., The influence of clothing on the physiological reactions of the human body to varying environmental temperatures. Am. J. Physiol., 1938, 124, 30.

8. Robinson, S., Turrell, E. S., and Gerking, S. D., Physiologically equivalent conditions of air temperature and humidity. Am. J. Physiol., 1945, 143, 21.

9. Hardy, J. D., and DuBois, E. F., The technique of measuring radiation and convection. J. Nutrition, 1938, 15, 461.

10. Borden, D. L., Waddill, J. F., and Grier, G. S., Statistical study of 265 cases of heat disease. J. A. M. A., 1945, 128, 1200.

11. Von Schlichtegroll, G., Zut Beurteilung der Arbeitsbedingungen bei hohen temperature. Arch. f. Gewerbepath. u. Gewerbehyg., 1941, 10, 575.

12. Horvath, S. M., and Shelley, W. B., Acclimatization to extreme heat and its effect on the ability to work in less severe environments. Am. J. Physiol., In Press. 